\title{
Co-existence of Beckwith-Wiedemann syndrome and autism spectrum disorder
}

\author{
Alper Alnak ${ }^{1 \oplus}$, Dilara Bulanik Ozdemirci ${ }^{\circledR}$, Murat Coskun $^{1 \oplus}$
}

${ }^{1}$ Istanbul University, Istanbul Faculty of Medicine, Department of Child and Adolescent Psychiatry, Istanbul - Turkey

\begin{abstract}
Autism spectrum disorder (ASD) is an early-onset neurodevelopmental disorder characterized by deficits in communicative and social skills and repetitive behaviors and/or restricted interests. The genetic mechanism underlying ASD is as complex and heterogeneous as its clinical heterogeneity. Beckwith-Wiedemann syndrome (BWS) is a well-known human imprinting disorder characterized by macroglossia, visceromegaly, hemihypertrophy, abdominal wall defects and a number of other various developmental abnormalities with an increased risk for embryonal tumors. In the literature, a greater frequency of neurobehavioral issues including ASD has been reported in children with BWS. Here, we present a 7-year-old boy diagnosed with ASD and BWS admitted to our clinic with complaints of emotional and behavioral changes after suddenly losing his father due to myocardial infarction. Upon initial assessment and psychiatric examination, the ASD diagnosis was confirmed and the exacerbation of the symptoms was assumed to be related with his bereavement. The family was given psychoeducation and the boy was started on escitalopram $0.5 \mathrm{mg} /$ day, gradually increased to $2.5 \mathrm{mg} /$ day. One month after this intervention, the family reported significant improvements in his symptoms. Although BWS has not been studied as extensively as other genetic conditions associated with neurodevelopmental disorders, it may be important for us to expand our knowledge about ASD accompanying human imprinting diseases including BWS.
\end{abstract}

Keywords: Autism, Beckwith-Wiedemann syndrome, imprinting disorder, overgrowth

\section{INTRODUCTION}

Autism spectrum disorder (ASD) is a neurodevelopmental disorder with multiple genetic factors contributing to its etiology in a complex and heterogeneous fashion. In some cases, ASD may be seen in conjunction with another syndromic disease (1). In most of these cases, an underlying etiology can be identified that involves chromosomal abnormalities, submicroscopic cytogenetic changes, and single gene mutations. The term "syndromic" ASD refers to this subpopulation with dysmorphic features and/or accompanying other phenotypes (2). Syndromic diseases constitute approximately $5 \%$ of all cases with ASD (3); syndromes such as Fragile X syndrome, Rett syndrome, Tuberous sclerosis, and Angelman syndrome are reported more frequently (4). It may be important to expand our knowledge about syndromic ASD, as it may have a different course and/or phenomenology than non-syndromic ASD (3). Furthermore, understanding the pathogenetic mechanisms at the level of molecular pathways and synaptic functioning may provide insights helping to understand the pathophysiology of non-syndromic

How to cite this article: Alnak A, Bulanik Ozdemirci D, Coskun M. Co-existence of Beckwith-Wiedemann syndrome and autism spectrum disorder. Dusunen Adam The Journal of Psychiatry and Neurological Sciences 2019;32:268-270.

Correspondence: Alper Alnak, Istanbul University, Istanbul Faculty of Medicine, Department of Child and Adolescent Psychiatry, Istanbul - Turkey Phone: +90 21241420 00/12885 E-mail: alperalnak@gmail.com 
ASD (3). Beckwith-Wiedemann syndrome (BWS) is a well-known human imprinting disorder (expression of gene according to its paternal allele) (5). A literature review revealed few reports of an increased risk of ASD in children with BWS (6). Here we present a child with ASD and BWS admitted to the outpatient clinic with complaints of behavioral disturbances following the loss of his father. His depressive symptoms were successfully treated with escitalopram. Here we discuss the co-existence of BWS and ASD as well as the management of depressive symptoms.

\section{CASE}

A 7-year-old male was brought to the Child and Adolescent Psychiatry Outpatient Clinic by his mother with complaints of hyperactivity, aggression, and increased repetitive behavior such as clapping, flapping, and vigorously hitting the floor with his hands. He had been diagnosed with BWS and ASD and had been in special education since he was 2 years old. His mother reported that those complaints worsened, and he had started behaving unusually for the last 3 months after the sudden loss of his father due to myocardial infarction. He was less happy than usual and seemed to show less interest in watching his favorite cartoons, playing with play dough, and going for walks. He was also frequently hitting his own head, had sleep disturbances, and developed bursts of crying just after the loss. The mother also stated that the body was not attending school regularly. He had been on risperidone treatment $(1 \mathrm{mg} /$ day $)$ for 1 year for his behavioral problems. Psychiatric examination of the patient revealed severe impairment in socialemotional reciprocity, limited non-verbal communication with poor eye contact, and absence of verbal expression other than a few meaningless words. He showed self-mutilative behaviors such as hitting his head and was preoccupied with his stereotypies. His medical history was remarkable for BWS, epilepsy, cryptorchidism, and patent foramen ovale. Upon initial assessment and psychiatric examination, his ASD diagnosis was confirmed and the exacerbation of his symptoms ascribed to his bereavement. Child neurology was consulted, and the family was given psychoeducation. After consultation, the patient was started on escitalopram $0.5 \mathrm{mg} / \mathrm{day}$, gradually increased to $2.5 \mathrm{mg} / \mathrm{day}$, while his risperidone dose remained unchanged. One month after this intervention, the family reported significant improvements in his symptoms. During the eight- months follow-up, improvement in other symptoms was recorded.

\section{DISCUSSION}

BWS is an overgrowth syndrome linked with abnormal expression of imprinted gene loci at $11 \mathrm{p}$ 15.5 that contains genes playing a crucial role in growth and growth suppression. Other overgrowth syndromes such as Fragile $\mathrm{X}$ (while not being an imprinting disorder) are shown to be associated with ASD (7). Other imprinting diseases and related imprinted genes, such as Angelman syndrome and Prader Willi syndrome, have been shown to be linked to different neuropsychiatric disorders including ASD (3). In comparison, less is known about BWS. Generally, it is thought that children with BWS will undergo normal neurodevelopment except in cases of coexisting chromosomal abnormality or perinatal hypoxic conditions (8). In the literature, increased frequency of neurobehavioral problems including ASD traits have been reported in children with BWS (8). In a study investigating behavioral phenotypegenotype in BWS conducted by Kent et al. (6) the prevalence of ASD in subjects with BWS was found to be $6.8 \%$. As in BWS, increased head circumference and higher levels of IGF2 are reported to be more common in children with ASD than in normally developing children. Nevertheless, the co-occurrence of these two diseases is poorly investigated in the literature and their relationship remains unclear. Worsening or emergence of behavioral/emotional problems following the loss of the father may be another clinical implication in this case. Children with ASD may go through bereavement and/or depression following the loss of loved ones, presenting differently than healthy children (9). Not only the loss of a loved one, but also disruptions to children's daily routine tasks may provoke distress. Children with a higher IQ and better verbal skills may understand and describe their feelings better, but children with lower social skills may show various behavioral/emotional problems including bursts of crying, social withdrawal, increase in stereotypic behaviors, decline in functioning, irritability, and sleep problems, as was the case in our patient. His behavioral and emotional problems were treated successfully with risperidone and escitalopram. Risperidone has been reported to be effective and well tolerated in treating behavioral problems in young children (10) and escitalopram at low doses was reported as safe and effective in the 
treatment of young children with emotional problems (11). Because young subjects with ASD may be more susceptible to side effects of psychiatric medications, starting at a low dose and slow titration is generally recommended (12).

It seems important for clinicians serving in child psychiatry clinics as well as other clinicians working with children to increase their awareness of neurodevelopmental disorders that may be seen in conjunction with other syndromic diseases for early risk assessment and interventions. Although BWS has not been studied as extensively as other genetic conditions associated with neurodevelopmental disorders, it may be important for us to expand our knowledge about ASD accompanying BWS and other human imprinting diseases in order to encourage studies shedding more light on their possible shared pathogenesis and treatment for these debilitating disorders.

\begin{tabular}{|c|c|c|}
\hline \multicolumn{2}{|c|}{ Contribution Categories } & Author Initials \\
\hline \multirow{4}{*}{ Category 1} & Concept/Design & M.C. \\
\hline & Literature review & D.B.O. \\
\hline & Data analysis/Interpretation & A.A. \\
\hline & Case follow-up (if applicable) & D.B.O. \\
\hline \multirow{2}{*}{ Category 2} & Drafting manuscript & M.C., A.A. \\
\hline & Critical revision of manuscript & M.C. \\
\hline Category 3 & Final approval and accountability & M.C. \\
\hline \multirow{2}{*}{ Other } & Technical or material support & M.C., A.A. \\
\hline & Supervision & M.C., D.B.O. \\
\hline
\end{tabular}

Acknowledgements: The authors would like to thank the patient and his family for their consent and collaboration.

Informed Consent: Written informed consent was obtained from the patient's parent for the publication of the case report.

Peer-review: Externally peer-reviewed.

Conflict of Interest: The authors declare that they have no conflict of interest.

Financial Disclosure: The authors declare no financial support.

\section{REFERENCES}

1. Karayagmurlu A, Gokcen C, Varan C. Morning glory syndrome and autism: a case report. Dusunen Adam The Journal of Psychiatry and Neurological Sciences 2015; 28:167-170.

2. Fernandez BA, Scherer SW. Syndromic autism spectrum disorders: moving from a clinically defined to a molecularly defined approach. Dialogues Clin Neurosci 2017; 19:353-371.

3. Sztainberg Y, Zoghbi HY. Lessons learned from studying syndromic autism spectrum disorders. Nat Neurosci 2016; 19:1408-1418.

4. Caglayan AO. Genetic causes of syndromic and non-syndromic autism. Dev Med Child Neurol 2010; 52:130-138.

5. Elliott M, Maher ER. Beckwith-Wiedemann syndrome. J Med Genet 1994; 31:560-564.

6. Kent L, Bowdin S, Kirby GA, Cooper WN, Maher ER. Beckwith Weidemann [sic] syndrome: A behavioral phenotype-genotype study. Am J Med Genet B Neuropsychiatr Genet 2008; 147B:1295-1297.

7. Harris SW, Hessl D, Goodlin-Jones B, Ferranti J, Bacalman S, Barbato I, Tassone F, Hagerman PJ, Herman K, Hagerman RJ. Autism profiles of males with Fragile X Syndrome. Am J Ment Retard 2008; 113:427-438.

8. Shuman C, Beckwith JB, Weksberg R. Beckwith-Wiedemann Syndrome. [Updated 2016 Aug 11]: In Adam MP, Ardinger HH, Pagon RA, Wallace SE, Bean LJH, Stephens K, Amemiya A. (editors). GeneReviews ${ }^{\circledR}$. Seattle (WA): University of Washington, 1993-2019. https://www.ncbi.nlm.nih.gov/books/ NBK1394. Accessed May 31, 2019.

9. Ghaziuddin M, Ghaziuddin N, Greden J. Depression in persons with autism: implications for research and clinical care. J Autism Dev Disord 2002; 32:299-306.

10. Coskun M, Zoroglu SS, Ozturk M. Risperidone treatment in preschool children with disruptive behavior disorders: a chart review study. Bulletin of Clinical Psychopharmacology 2011; 21:33-41.

11. Coskun M, Ozturk M, Zoroglu S. Escitalopram treatment in preschool children with anxiety disorders: a case series. Bulletin of Clinical Psychopharmacology 2012; 22:262-267.

12. Owley T, Walton L, Salt J, Guter SJ, Winnega M, Leventhal BL, Cook EH Jr. An open-label trial of escitalopram in pervasive developmental disorders. J Am Acad Child Adolesc Psychiatry 2005; 44:343-348. 